Pacific Journal of Mathematic 


\section{TRANSFORMATIONS OF SYMMETRIC TENSORS}

\section{LARRY CUMmINGS}

This paper is about linear transformations of the $k$-fold symmetric tensor product of an $n$-dimensional vector space $V$ which carry nonzero decomposable tensors to nonzero decomposable tensors. The main theorem shows that every such transformation is induced by a nonsingular transformation of $V$ provided both

(i) the field has characteristic either 0 or a prime greater than $k$ and every polynomial over the field with degree at $n$ is a product of linear factors.

(ii) $n>k+1$.

Condition (i) includes the important special case where the field is algebraically closed with characteristic 0 .

The linear transformations which preserve decomposable tensors in the skew-symmetric case have been studied in two papers by Westwick [6, 8]. In [6] he showed that if the field is algebraically closed then the transformation is induced by a linear transformation of $V$ except, possibly, when the dimension of $V$ is $2 k$. In the latter case the transformation may be the composition of one induced by a linear transformation of $V$ and one induced by a correlation of the $k$-dimensional subspaces of $V$. A series of papers $[3,4,7,2]$ has been devoted to linear transformations which preserve decomposable tensors in the case of the full tensor product.

Our result partially answers a question first raised by Marcus and Newman in [5]. They asked for necessary and sufficient conditions in order that every decomposable mapping of the space of $k$-fold symmetric tensors be induced.

1. Preliminaries. Let $V^{k}$ denote the $k$-fold Cartesian product of $V$ where $k>1$. A $k$-fold symmetric tensor space (or rank $k$ symmetric tensor space) is a vector space denoted by $V_{k} V$ together with a fixed multilinear symmetric mapping $\sigma: V^{k} \rightarrow \mathrm{V}_{k} V$ which is universal for multilinear and symmetric mappings of $\mathrm{V}_{k} V$. We assume that $\mathrm{V}_{k} V$ is generated by the image of $\sigma$. Thus, if $W$ is any vector space and $g: V^{k} \rightarrow W$ is both multilinear and symmetric then $g$ has a unique extension $h: \mathbf{V}_{k} V \rightarrow W$ such that

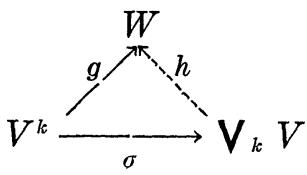


is commutative and $\mathrm{V}_{k} V$ is isomorphic to any other vector space with this property. In particular, if $A: V \rightarrow V$ is linear then the assignment

$$
\left(x_{1}, \cdots, x_{k}\right) \longmapsto A x_{1} \vee \cdots \vee A x_{k}
$$

is a multilinear and symmetric mapping of $V^{k}$. We will denote its unique linear extension to $\mathrm{V}_{k} V$ by $\mathrm{V}_{k} A$.

The decomposable symmetric tensors or "symmetric products" are images under $\sigma$ of $k$-tuples in $V^{k}$. For convenience we denote $\sigma\left(x_{1}, \cdots, x_{k}\right)$ by $x_{1} \vee \cdots \vee x_{k}$. A subspace $s$ of $\mathrm{V}_{k} V$ is decomposable if $S \subseteq \sigma\left(V^{k}\right)$. Trivial decomposable subspaces are the zero subspace and the 1-dimensional subspaces whose elements are scalar multiples of a single nonzero decomposable symmetric tensor. If $V$ and $F$ satisfy (i) and (ii) the maximal decomposable subspaces of $\mathrm{V}_{k} V$ were determined in [1].

A symmetric product is zero if and only if at least one of its factors is zero. More generally, if

$$
x_{1} \vee \cdots \vee x_{k}=y_{1} \vee \cdots \vee y_{k} \neq 0
$$

then there are scalars $\lambda_{1}, \cdots, \lambda_{k}$ such that $\lambda_{1} \cdots \lambda_{k}=1$ and

$$
x_{i}=\lambda_{i} y_{\pi(i)} \quad i=1, \cdots, k .
$$

Here $\pi \in S_{k}$, the symmetric group on $\{1, \cdots, k\}$.

A linear transformation $f: \mathbf{V}_{k} V \rightarrow \mathbf{V}_{k} V$ is decomposable if

$$
f\left(\sigma\left(V^{k}\right)\right) \cong \sigma\left(V^{k}\right)
$$

and

$$
\operatorname{ker} f \cap \sigma\left(V^{k}\right)=0 \text {. }
$$

If $V$ is an $n$-dimensional vector space then the dimension of $\mathrm{V}_{k} V$ is $\left(\begin{array}{c}n+k-1 \\ k\end{array}\right)$.

2. Type 1 subspaces and associate mappings. Subspaces in $\mathrm{V}_{k} V$ of the form

$$
M=x_{1} \vee \cdots \vee x_{k-1} \vee V
$$$$
k>1
$$

where $x_{1}, \cdots, x_{k-1}$ are fixed nonzero vectors in $V$ are always decomposable because of the multilinearity of the mapping $\sigma$. It is convenient to call these type 1 subspaces. The 1-dimensional subspaces $\left\langle x_{1}\right\rangle, \cdots,\left\langle x_{k-1}\right\rangle$ are called the factors of $M$.

Proposition 1. If $F$ is a field whose characteristic (if any) is 
not less than $k$ then

$$
x_{1} \vee \cdots \vee x_{k-1} \vee V=x_{1}^{\prime} \vee \cdots \vee x_{k-1}^{\prime} \vee V \quad \text { in } \mathrm{V}_{k} V
$$

implies

$$
\left\langle x_{1} \vee \cdots \vee x_{k-1}\right\rangle=\left\langle x_{1}^{\prime} \vee \cdots \vee x_{k-1}^{\prime}\right\rangle \quad \text { in } \bigvee_{k-1} V
$$

Proof. This proof requires the choice of a vector not in the set-theoretic union

$$
\left\langle x_{1}\right\rangle \cup \cdots \cup\left\langle x_{k-1}\right\rangle \text {. }
$$

By Lemma 12 of [1, p. 73] we know that if $V$ were the union (2.3) then the cardinality of $F$ could not exceed the finite integer $k-1$. This would mean that the characteristic of $F$ exceeds the cardinality of $F$. Accordingly we may choose $v$ in $V$ not in the union (2.3) and (2.2) implies the existence of a $u$ in $V$ satisfying

$$
x_{1} \vee \cdots \vee x_{k-1} \vee u=x_{1}^{\prime} \vee \cdots \vee x_{k-1}^{\prime} \vee v \text {. }
$$

By the choice of $v$ and (1.2) there is a nonzero scalar $\lambda$ for which $u=\lambda v$ and

$$
x_{i}=\lambda_{i} x_{\pi(i)}^{\prime} \quad i-1, \cdots, k-1
$$

where $\pi \in S_{k-1}$ and $1=\lambda \Pi \lambda_{i}$. Therefore,

$$
\lambda x_{1} \vee \cdots \vee x_{k-1}=x_{1}^{\prime} \vee \cdots \vee x_{k-1}^{\prime}
$$

in $\mathbf{V}_{k-1} V$.

Hereafter we will assume that $F$ satisfies the hypothesis of Proposition 1.

A type 1 mapping is a decomposable mapping of $\mathbf{V}_{k} V$ for which the image of every type 1 subspace is again a type 1 subspace. If $f$ is a type 1 mapping and $M$ is the type 1 subspace (2.1) then we may choose nonzero vectors $y_{1}, \cdots, y_{k-1}$ in $V$ such that

$$
f(M)=y_{1} \vee \cdots \vee y_{k-1} \vee V \text {. }
$$

We obtain a well-defined linear mapping $A$ of $V$ by setting $A u=v$ if

$$
f\left(x_{1} \vee \cdots \vee x_{k-1} \vee u\right)=y_{1} \vee \cdots \vee y_{k-1} \vee v .
$$

The mapping $A$ will be called an associate mapping of $f$ with respect to $M$. In general, the associate map defined by (2.5) depends not only on $M$ and $f$ but the choice of the vectors $y_{1}, \cdots, y_{k-1}$ as well. 
Proposition 2. Any two associate mappings of a type 1 mapping with respect to the same type 1 subspace are multiples.

Proof. This follows easily from Proposition 1 and (1.1).

Proposition 3. Every associate of a type 1 mapping is nonsingular.

Proof. Let $A$ be an associate of a type 1 mapping $f$ with respect to (2.1) and suppose $A(u)=A\left(u^{\prime}\right)$ for some vectors $u, u^{\prime}$ in $V$. From (2.5) we have

$$
f\left(x_{1} \vee \cdots \vee x_{k-1} \vee u\right)=f\left(x_{1} \vee \cdots \vee x_{k-1} \vee u^{\prime}\right) .
$$

Since $f$ is linear and decomposable we have

$$
x_{1} \vee \cdots \vee x_{k-1} \vee\left(u-u^{\prime}\right)=0
$$

which implies $u=u^{\prime}$.

Two type 1 subspaces will be called adjacent if they have exactly $k-2$ common factors (counting multiplicity). Accordingly a typical pair of adjacent subspaces may be written in the form

$$
M_{i}=x_{1} \vee \cdots \vee x_{k-1} \vee z_{i} \vee V \quad i=1,2
$$

where $z_{1}, z_{2}$ are two independent vectors of $V$ and $x_{1}, \cdots, x_{k-1}$ are arbitrary nonzero vectors.

Two arbitrary type 1 subspaces are always connected by a chain of adjacent subspaces; explicitly, if

$$
M=x_{1} \vee \cdots \vee x_{k-1} \vee V
$$

and

$$
N=y_{1} \vee \cdots \vee y_{k-1} \vee V
$$

then $M_{p}$ is adjacent to $M_{p+1}$ where

$$
M_{p}=x_{1} \vee \cdots \vee x_{k-p-1} \vee y_{1} \vee \cdots \vee y_{p} \vee V \quad p=1, \cdots, k-2
$$

and we take $M=M_{0}$ and $N=M_{k-1}$.

Proposition 4. Two type 1 subspaces $M$ and $N$ are adjacent if and only if $\operatorname{dim} M \cap N=1$. Otherwise $M \cap N=0$ whenever $M$ and $N$ are distinct.

Proof. Consider the adjacent type 1 subspaces (2.6). If $t \in M_{1} \cap M_{2}$ then there exist vectors $u$ and $v$ in $V$ such that 


$$
t=x_{1} \vee \cdots \vee x_{k-2} \vee z_{1} \vee u=x_{1} \vee \cdots x_{k-2} \vee z_{2} \vee v
$$

Now the multilinear and symmetric mapping $g_{p}(x): V^{p} \rightarrow \mathbf{V}_{p+1} V$ defined for each $p=2, \cdots, k-1$ by

$$
\left(v_{1}, \cdots, v_{p}\right) \longmapsto x \vee v_{1} \vee \cdots \vee v_{p}
$$

extends as in (1.1) to a linear mapping $h_{p}(x): \mathrm{V}_{p} V \rightarrow \mathrm{V}_{p+1} V$. If the vector $x$ in (2.10) is nonzero then each $h_{p}(x)$ is injective and so is the composite

$$
h=h_{k-1}\left(x_{1}\right) \cdots h_{k-i}\left(x_{i}\right) \cdots h_{2}\left(x_{k-2}\right) .
$$

Thus (2.9) is just

$$
h\left(z_{1} \vee u\right)=h\left(z_{2} \vee v\right)
$$

and so

$$
z_{1} \vee u=z_{2} \vee v \text {. }
$$

Since $z_{1}$ and $z_{2}$ are independent (1.2) implies that $u$ is a scalar multiple of $z_{2}$. Therefore

$$
M_{1} \cap M_{2}=\left\langle x_{1} \vee \cdots \vee x_{k-2} \vee z_{1} \vee z_{2}\right\rangle .
$$

Now consider an arbitrary pair of type 1 subspaces (2.7) and suppose they have nonzero intersection. Let

$$
t=x_{1} \vee \cdots \vee x_{k-1} \vee u=y_{1} \vee \cdots \vee y_{k-1} \vee v
$$

be a nonzero element of the intersection. If $\langle u\rangle=\langle v\rangle$ then by (1.2) we have $M_{1}=M_{2}$ and otherwise $M_{1}$ and $M_{2}$ must have exactly $k-2$ common factors.

Proposition 5. The images of adjacent type 1 subspaces under type 1 mappings are adjacent provided the underlying field satisfies (i).

Proof. Consider the adjacent type 1 subspaces (2.6). We know from Proposition 4 that

$$
M_{1} \cap M_{2}=\left\langle x_{1} \vee \cdots \vee x_{k-2} \vee z_{1} \vee z_{2}\right\rangle \cdot
$$

If $f$ is a type 1 mapping then $f\left(M_{1}\right) \cap f\left(M_{2}\right)$ is nonzero and Proposition 4 yields the desired conclusion provided $f\left(M_{1}\right)$ and $f\left(M_{2}\right)$ are distinct. We complete the proof by showing that the images of adjacent subspaces are always distinct.

Consider the two linear mappings $A_{i}: V \rightarrow \mathrm{V}_{k} V$ defined by 


$$
A_{i}(v)=f\left(x_{1} \vee \cdots \vee x_{k-1} \vee z_{i} \vee v\right) \quad i=1,2 .
$$

It follows that they are injective because $f$ is linear and decomposable. Suppose range $A_{1}=$ range $A_{2}$ and let $A_{2}^{-1}$ : range $A_{2} \rightarrow V$ be the inverse of $A_{2}$. Then $A_{2}^{-1} A_{1}$ is a well-defined linear transformation of $V$. Because of (i), $A_{2}^{-1} A_{1}$ has at least one characteristic value, say $\lambda$. If $u$ is a corresponding characteristic vector then $A_{1} u=\lambda A_{2} u$. That is,

$$
f\left(x_{1} \vee \cdots \vee x_{k-1} \vee z_{1} \vee u\right)=\lambda f\left(x_{1} \vee \cdots \vee x_{k-1} \vee z_{2} \vee u\right) .
$$

Since $f$ is linear and decomposable we obtain $z_{1}=\lambda z_{2}$, contradicting the assumption that $M_{1}$ and $M_{2}$ are adjacent.

Any collection of two or more type 1 subspaces in $\mathrm{V}_{k} V(k>2)$ will be called an adjacent family if there are vectors $x_{1}, \cdots, x_{k-2}$ in $V$ such that any subspace in the collection can be written as

$$
x_{1} \vee \cdots \vee x_{k-2} \vee u \vee V
$$

for some vector $u \in V$. When $k=2$ any collection containing at least two distinct type 1 subspaces will be called an adjacent family. Of course every pair of adjacent type 1 subspaces constitutes an adjacent family, but a collection of three or more need not be, as is easily seen by example.

Proposition 6. Any collection of more than $k$ pair-wise adjacent type 1 subspaces in $\mathbf{V}_{k} V$ is an adjacent family.

Proof. We assign to each type 1 subspace (2.1) the set

$$
\left\{\left(\left\langle x_{i}\right\rangle, i\right) \mid i=1, \cdots, k-1\right\}
$$

which always contains $k-1$ distinct elements even if (2.1) does not have distinct factors.

The proposition now follows from the combinatorial result that a collection of more than $k$ finite sets each containing $k-1$ elements which intersect pair-wise in $k-2$ elements always intersect in the same set of $k-2$ elements:

If $k=2$ there is nothing to prove. If $k>2$ let $X$ and $Y$ be any two sets of the collection. There are elements $a$ and $b$ such that

$$
X=(X \cap Y) \cup\{a\}
$$

and

$$
Y=(X \cap Y) \cup\{b\} .
$$


Because any two sets in the collection intersect in $k-2$ elements, any set of the collection not containing $X \cap Y$ must contain both $a$ and $b$ and intersect $X \cap Y$ in exactly $k-3$ elements. But there are at most $k-2=\left(\begin{array}{l}k-2 \\ k-3\end{array}\right)$ distinct such sets. Therefore, the collection must contain at least one set $Z$ distinct from $X$ and $Y$ but which contains $X \cap Y$. Let

$$
Z=X \cap Y \cup\{c\}
$$

and suppose there exists a set $W$ in the collection not containing $X \cap Y$. Then $\{a, b, c\} \subseteq W$, contradicting the hypothesis that $X \cap W$ has $k-2$ elements.

3. Main results. A collection of vectors in an $n$-dimentional vector space is said to be in general position when any $n$ vectors chosen from the collection form a basis of $V$. The following well known lemma about vectors in general position will be used in showing that any two associate mappings of a type 1 mapping are multiples whenever $n>2$ and the underlying field is infinite.

LEMMA 1. If $m \geqq n$ then an $n$-dimensional vector space over an infinite field always contains $m$ vectors in general position.

Lemma 2. Let $z_{1}, \cdots, z_{m}$ be any finite set of vectors in an $n$ dimensional vector space over an infinite field. If $A: V \rightarrow V$ is nonsingular and $B$ is any other linear mapping of $V$ satisfying

$$
\langle A(x)\rangle=\langle B(x)\rangle
$$

for all vectors $x$ not in $S=\left\langle z_{1}\right\rangle \cup \cdots \cup\left\langle z_{m}\right\rangle$ then there is a scalar $\lambda$ such that $B=\lambda A$.

Proof. Since $F$ is infinite Lemma 12 of [1] and induction show the existence of a basis of $V$ disjoint from the set $S$. If $b_{1}, \cdots, b_{n}$ is such a basis let $\lambda_{1}, \cdots, \lambda_{n}$ be scalars such that

$$
B\left(b_{i}\right)=\lambda_{i} A\left(b_{i}\right) \quad i=1, \cdots, n .
$$

Since $F$ is infinite we may choose a vector $v=\Sigma \alpha_{i} b_{i}$ not in $S$ but all of whose coordinates with respect to $b_{1}, \cdots, b_{n}$ are non-zero. Then (3.1) and (3.2) imply the existence of a scalar $\lambda$ such that

$$
\Sigma \alpha_{i} \lambda_{i} A\left(b_{i}\right)=\Sigma \lambda \alpha_{i} A\left(b_{i}\right) \text {. }
$$

Since $A$ is nonsingular we have $\lambda_{1}=\lambda_{2}=\cdots=\lambda_{n}=\lambda$.

REMARK. In (i) we assume that every polynomial of degree at 
most $n$ splits completely over the underlying field. This means that the field is necessarily infinite since the polynomial ring over a finite field has irreducible elements of every degree. Thus Lemmas 1 and 2 are immediately applicable in the following theorems.

THEOREM 1. The associate mappings of a type 1 mapping of $\mathrm{V} k V$ are a 1-dimensional subspace of the linear mappings of $V$, provided $\operatorname{dim} V>2$ and $F$ satisfies (i).

Proof. We show first that an associate map of a type 1 mapping $f$ with respect to one of type 1 subspaces (2.6) is always a scalar multiple of every associate mapping of the other. By Lemma 1 we complete the vectors $z_{1}, z_{2}$ to a set $z_{1}, \cdots, z_{m}$ in general position where $m=\operatorname{Max}\{k, \operatorname{dim} V\}$. As in the proof of the Proposition 1 we may choose a vector $z_{m+1}$ not in the set-theoretic union $\left\langle z_{1}\right\rangle \cup \cdots \cup\left\langle z_{m}\right\rangle$. Then the subspaces

$$
M_{i}=x_{1} \vee \cdots \vee x_{k-2} \vee z_{i} \vee V \quad i=1, \cdots, m+1
$$

are an adjacent family. The images of these subspaces form a family of pair-wise adjacent subspaces by Proposition 5. They form an adjacent family by Proposition 6 and the choice of $m$. Thus we may choose vectors $y_{1}, \cdots, y_{k-2} ; w_{1}, \cdots, w_{m+1}$ in $V$ such that

$$
f\left(M_{i}\right)=y_{1} \vee \cdots \vee y_{k-2} \vee w_{i} \vee V \quad i=1, \cdots, m+1 .
$$

We proceed to examine the effect of $f$ on the intersections $M_{i} \cap M_{m+1} ; i=1,2$. By (3.3)

$$
\begin{array}{r}
f\left(x_{1} \vee \cdots \vee x_{k-2} \vee z_{i} \vee z_{m+1}\right)= \\
=y_{1} \vee \cdots \vee y_{k-2} \vee w_{i} \vee A_{i}\left(z_{m+1}\right) \\
=y_{1} \vee \cdots \vee y_{k-2} \vee w_{m+1} \vee A_{m+1}\left(z_{i}\right) \\
i=1,2 .
\end{array}
$$

where $A_{i}$ denotes any associate map of $M_{i}$ under $f$ and $A_{m+1}$ is an associate of $M_{m+1}$. It follows that $\left\langle w_{m+1}\right\rangle=\left\langle A_{i}\left(z_{m+1}\right)\right\rangle$ for $i=1,2$ because $w_{m+1}$ is not in $\left\langle w_{1}\right\rangle \cup\left\langle w_{2}\right\rangle$. Since $z_{m+1}$ is restricted only by its exclusion from $\left\langle z_{1}\right\rangle \cup \cdots \cup\left\langle z_{m}\right\rangle$ Lemma 2 applies and yields a scalar $\gamma$ such that $A_{1}=\gamma A_{2}$.

To complete the proof we need only consider an arbitrary pair of type 1 subspaces (2.7) and a chain (2.8) of adjacent subspaces between them. If $A_{p}$ is an associate map of $M_{p}$ then we have just shown the existence of a scalar $\gamma_{p}$ such that

$$
A_{p}=\gamma_{p} A_{p+1} \quad p=0, \cdots, k-2 .
$$

Therefore, $A_{0}=\gamma_{0} \cdots \gamma_{k-2} A_{k-1}$. 
REMARK. If $\operatorname{dim} V=1$ then $\mathbf{\bigvee}_{k} V=1$ and $L\left(\mathbf{V}_{k} V, \mathbf{V}_{k} V\right) \cong F$. Hence $L\left(\mathrm{~V}_{k} V, \mathrm{~V}_{k} V\right)$ consists of induced mappings if and only if every polynomial of the form $x^{k}-a$ has a root in $F$.

THEOREM 2. Every type 1 mapping of $\mathbf{V}_{k} V$ is induced by an associate mapping, provided $\operatorname{dim} V>2$ and $F$ satisfies (i).

Proof. Let $x=x_{1} \vee \cdots \vee x_{k}$ be any nonzero product of $V_{k} V$. The trivial subspace $\langle x\rangle$ is the intersection of the $k$ type 1 subspaces

$$
T_{i}=x_{1} \vee \cdots \vee \hat{x}_{i} \vee \cdots \vee x_{k} \vee V \quad i=1, \cdots, k .
$$

By Theorem 1 the associate mappings of a type 1 mapping $f$ with respect to the subspaces (3.4) are scalar multiples of one another. If $A$ is any one of them then Theorem 1 and definition (2.5) show then that $A x_{i}$ must be a factor of $f(x)$ for each $i=1, \cdots, k$. Thus, if $x$ has distinct factors it follows from (1.2) and Proposition 3 that

$$
f(x)=\lambda_{x} A x_{1} \vee \cdots \vee A x_{k}
$$

for some scalar $\lambda_{x}$ and

$$
f\left(T_{i}\right)=A x_{1} \vee \cdots \vee \widehat{A x_{i}} \vee \cdots \vee A x_{k} \vee V \quad i=1, \cdots, k .
$$

We next verify (3.6) when the factors $\left\langle x_{1}\right\rangle, \cdots,\left\langle x_{k}\right\rangle$ are not necessarily distinct. To this end consider a chain of adjacent subspaces (2.8) where we suppose $M_{k-1}$ has arbitrary factors and take the factors of $M_{0}$ as distinct and distinct from the factors of $M_{k-1}$. This we may always do since any field satisfying (i) must be infinite. (See the remark following Lemma 2.) Thus (3.6) may be applied to $M_{0}$ which contains $z_{1}=x_{1} \vee \cdots \vee x_{k-1} \vee y_{1}$. By Theorem 1 there is a scalar $\lambda$ for which

$$
f\left(z_{1}\right)=\lambda A x_{1} \vee \cdots \vee A x_{k-1} \vee A y_{1} .
$$

Therefore the $k-1$ factors of $f\left(M_{1}\right)$ must be among the factors of (3.7). Now $\left\langle A y_{1}\right\rangle$ could not be excluded because then $M_{0}$ and $M_{1}$ would have the same type 1 subspace as image, contradicting Proposition 5. If, say, $A x_{1}$ were excluded then

$$
f\left(M_{1}\right)=A x_{2} \vee \cdots \vee A x_{k-1} \vee A y_{1} \vee V
$$

and Theorem 1 yields

$$
f\left(z_{1}\right)=\lambda_{1} A x_{2} \vee \cdots \vee A y_{1} \vee A x_{k-1}
$$

for some scalar $\lambda_{1}$.

Comparison of (3.7) and (3.8) shows that $A x_{k-1}$ would be a scalar 
multiple of either $A y_{1}$ or some $A x_{i}$ with $1 \leqq i<k-1$. Hence

$$
f\left(M_{1}\right)=A x_{1} \vee \cdots \vee A x_{k-2} \vee A y_{1} \vee V .
$$

Suppose it has been shown that

$$
f\left(M_{p}\right)=A x_{1} \vee \cdots \vee A x_{k-p-1} \vee A y_{1} \vee \cdots \vee A y_{p} \vee V
$$

for some $p, 1<p \leqq k-2$. Since

$$
M_{p} \cap M_{p+1}=\left\langle x_{1} \vee \cdots \vee x_{k-p-1} \vee y_{1} \vee \cdots \vee y_{p+1}\right\rangle
$$

(3.9) implies that $f\left(M_{p+1}\right)$ contains

$$
A x_{1} \vee \cdots \vee A x_{k-p-1} \vee A y_{1} \vee \cdots \vee A y_{p+1}
$$

and so the $k-1$ factors of $f\left(M_{p+1}\right)$ are among the factors of (3.10). Arguing as before we see that $A y_{p+1}$ must be a factor of $f\left(M_{p+1}\right)$ since otherwise the images of $f\left(M_{p}\right)$ and $f\left(M_{p+1}\right)$ would coincide. If, say, $A x_{1}$ were not a factor then

$$
f\left(M_{p+1}\right)=A x_{2} \vee \cdots \vee A x_{k-p-1} \vee A y_{1} \vee \cdots \vee A y_{p+1} \vee V
$$

and by Theorem 1 there is a scalar $\mu$ for which

$$
\begin{aligned}
& f\left(x_{1} \vee \cdots \vee x_{k-p-1} \vee y_{1} \vee \cdots \vee y_{p+1}\right) \\
= & \mu A x_{2} \vee \cdots \vee A x_{k-p-1} \vee A y_{1} \vee \cdots \vee A y_{p+1} \vee A x_{k-p-1} .
\end{aligned}
$$

Comparison of (3.10) and (3.11) shows that $A x_{k-p-1}$ would be either a multiple of some $A y_{i}, 1 \leqq i \leqq p+1$, or some $A x_{j}, 1 \leqq j<k-p-1$, contradicting the assumption that the factors of $M_{0}$ are distinct and distinct from the factors of $M_{k-1}$.

Since any product $x$ is in some type 1 subspace we have shown that $f(x)=\lambda_{x}\left(\mathrm{~V}_{k} A\right)(x)$ for some scalar $\lambda_{x}$. If $x$ and $y$ are products in the same type 1 subspace a simple comparison argument shows that $\lambda_{x}=\lambda_{y}$. Denote the common value by $\lambda$. When $x$ and $y$ are arbitrary products we obtain the same result by considering type 1 subspaces containing them and a chain (2.8) between the subspaces since any two of the latter have 1-dimensional intersections. Because the field always contains a root of $x^{k}-\lambda=0$ by (i), we have shown that $f$ is induced by $\lambda^{1 / k} A$.

THEOREM 3. Every decomposable mapping of $\mathbf{V}_{k} V$ is induced by a nonsingular mapping of $V$, provided $V$ is a finite dimensional vector space satisfying (i) and (ii).

Proof. Because of the previous theorem we need only show with the additional hypothesis that every decomposable mapping of 
$\mathrm{V}_{k} V$ is type 1 . If $M$ is any type 1 subspace and $f$ decomposable then $f(M)$ is a decomposable subspace and hence contained in a maximal decomposable subspace of $\mathbf{V}_{k} V$. In [1] the maximal decomposable subspaces of $\mathrm{V}_{k} V$ were determined for the case when $V$ satisfies the hypothesis of this theorem. The subspaces are

(a) type 1 subspaces

(b) type $r$ subspaces which are of the form

$$
x_{1} \vee \cdots \vee x_{k-r} \vee S \vee \cdots \vee S
$$

where $1<r \leqq k$ and $S$ is a 2-dimensional subspace of $V$.

Those subspaces of type $r>1$ have dimension $r+1$. If the maximal decomposable subspace containing $f(M)$ was one of these types then $\operatorname{dim} V \leqq r+1 \leqq k+1$ by (1.3) because every type 1 subspace has the same dimension as $V$. The hypothesis $\operatorname{dim} V>k+1$ thus implies that the maximal decomposable subspace containing $f(M)$ is type 1 and therefore $f$ is type 1 .

\section{REFERENCES}

1. L. J. Cummings, Decomposable symmetric tensors, Pacific J. Math., 35 (1970), 65-77.

2. D. Z̆. Djoković, Linear transformations of tensor products preserving a fixed rank, Pacific J. Math., 30 (1969), 411-414.

3. M. Marcus and B. M. Moyls, Linear transformations on algebras of matrices, Canad. J. Math., 11 (1959), 61-66.

4. - Transformations on tensor product spaces, Pacific J. Math., 9 (1959), 1215-1221.

5. M. Marcus and M. Newman, Inequalities for the permanent function, Ann. of Math., 75 (1962), 47-62.

6. R. Westwick, Linear transformations on Grassmann spaces, Pacific J. Math., 14 (1964), 1123-1127.

7. - Transformations on tensor spaces, Pacific J. Math., 23 (1967), 613-620.

8. - Linear transformations on Grassman spaces, Canad. J. Math., 21 (1969), $414-417$.

Received June 10, 1971 and in revised form October 22, 1971. Part of this work was contained in the author's thesis written under R. Westwick at the University of British Columbia. The author is also indebted to conversations with B. N. Moyls. 



\section{PACIFIC JOURNAL OF MATHEMATICS}

\section{EDITORS}

\section{H. SAMELSON}

Stanford University

Stanford, California 94305

\section{R. HOBBY}

University of Washington

Seattle, Washington 98105

\section{J. DugundjI}

Department of Mathematics University of Southern California Los Angeles, California 90007

RICHARD ARENS

University of California

Los Angeles, California 90024

\section{ASSOCIATE EDITORS}
E. F. BECKENBACH
B. H. NeumanN
F. WOLF
K. YOSHIDA

\section{SUPPORTING INSTITUTIONS}

\author{
UNIVERSITY OF BRITISH COLUMBIA \\ CALIFORNIA INSTITUTE OF TECHNOLOGY \\ UNIVERSITY OF CALIFORNIA \\ MONTANA STATE UNIVERSITY \\ UNIVERSITY OF NEVADA \\ NEW MEXICO STATE UNIVERSITY \\ OREGON STATE UNIVERSITY \\ UNIVERSITY OF OREGON \\ OSAKA UNIVERSITY
}

\author{
UNIVERSITY OF SOUTHERN CALIFORNIA \\ STANFORD UNIVERSITY \\ UNIVERSITY OF TOKYO \\ UNIVERSITY OF UTAH \\ WASHINGTON STATE UNIVERSITY \\ UNIVERSITY OF WASHINGTON \\ AMERICAN MATHEMATICAL SOCIETY \\ NAVAL WEAPONS CENTER
}

The Supporting Institutions listed above contribute to the cost of publication of this Journal, but they are not owners or publishers and have no responsibility for its content or policies.

Mathematical papers intended for publication in the Pacific Journal of Mathematics should be in typed form or offset-reproduced, (not dittoed), double spaced with large margins. Underline Greek letters in red, German in green, and script in blue. The first paragraph or two must be capable of being used separately as a synopsis of the entire paper. The editorial "we" must not be used in the synopsis, and items of the bibliography should not be cited there unless absolutely necessary, in which case they must be identified by author and Journal, rather than by item number. Manuscripts, in duplicate if possible, may be sent to any one of the four editors. Please classify according to the scheme of Math. Rev. Index to Vol. 39. All other communications to the editors should be addressed to the managing editor, Richard Arens, University of California, Los Angeles, California, 90024.

50 reprints are provided free for each article; additional copies may be obtained at cost in multiples of 50 .

The Pacific Journal of Mathematics is published monthly. Effective with Volume 16 the price per volume (3 numbers) is $\$ 8.00$; single issues, $\$ 3.00$. Special price for current issues to individual faculty members of supporting institutions and to individual members of the American Mathematical Society: $\$ 4.00$ per volume; single issues $\$ 1.50$. Back numbers are available.

Subscriptions, orders for back numbers, and changes of address should be sent to Pacific Journal of Mathematics, 103 Highland Boulevard, Berkeley, California, 94708.

PUBLISHED BY PACIFIC JOURNAL OF MATHEMATICS, A NON-PROFIT CORPORATION

Printed at Kokusai Bunken Insatsusha (International Academic Printing Co., Ltd.), 270, 3-chome Totsuka-cho, Shinjuku-ku, Tokyo 160, Japan. 


\section{Pacific Journal of Mathematics}

\section{Vol. 42, No. $3 \quad$ March, 1972}

Catherine Bandle, Extensions of an inequality by Pólya and Schiffer for vibrating membranes ................................ 543

S. J. Bernau, Topologies on structure spaces of lattice groups.......... 557

Woodrow Wilson Bledsoe and Charles Edward Wilks, On Borel product measures .......................................

Eggert Briem and Murali Rao, Normpreserving extensions in subspaces of

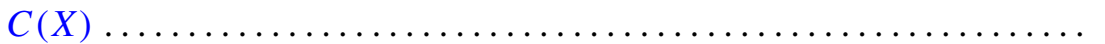

Alan Seymour Cover, Generalized continuation.................. 589

Larry Jean Cummings, Transformations of symmetric tensors .......... 603

Peter Michael Curran, Cohomology of finitely presented groups .......... 615

James B. Derr and N. P. Mukherjee, Generalized quasicenter and

hyperquasicenter of a finite group ...................... 621

Erik Maurice Ellentuck, Universal cosimple isols .................. 629

Benny Dan Evans, Boundary respecting maps of 3-mainfolds .......... 639

David F. Fraser, A probabilistic method for the rate of convergence to the

Dirichlet problem .................................. 657

Raymond Taylor Hoobler, Cohomology in the finite topology and Brauer

groups ..................................... 667

Louis Roberts Hunt, Locally holomorphic sets and the Levi form ........ 681

B. T. Y. Kwee, On absolute de la Vallée Poussin summability............ 689

Gérard Lallement, On nilpotency and residual finiteness in semigroups .... 693

George Edward Lang, Evaluation subgroups of factor spaces........... 701

Andy R. Magid, A separably closed ring with nonzero torsion pic ....... 711

Billy E. Rhoades, Commutants of some Hausdorff matrices ............. 715

Maxwell Alexander Rosenlicht, Canonical forms for local derivations . . . . 721

Cedric Felix Schubert, On a conjecture of L. B. Page ................ 733

Reinhard Schultz, Composition constructions on diffeomorphisms of $S^{p} \times S^{q}$

J. P. Singhal and H. M. (Hari Mohan) Srivastava, A class of bilateral generating functions for certain classical polynomials ....

Richard Alan Slocum, Using brick partitionings to establish conditions which insure that a Peano continuum is a 2-cell, a 2-sphere or an annulus...

James F. Smith, The p-classes of an $H^{*}$-algebra ...

Jack Williamson, Meromorphic functions with negative zeros and positive

poles and a theorem of Teichmuller ................. 